\title{
Chemical properties of field halo and thick disk blue straggler stars: first results
}

\author{
Angela Bragaglia ${ }^{1}$, Eugenio Carretta ${ }^{1}$, Alejandra Recio Blanco ${ }^{2}$, \\ Carla Cacciari ${ }^{1}$ and T.D. Kinman ${ }^{3}$ \\ ${ }^{1}$ INAF-Osservatorio Astronomico di Bologna, via Ranzani 1, 40127 Bologna (Italy) \\ email: angela.bragaglia eugenio.carretta carla.cacciari @bo.astro.it \\ ${ }^{2}$ Observatoire de la Côte d'Azur, Nice (France) \\ email: alejandra.recio-blanco@obs-nice.fr \\ ${ }^{3}$ NOAO, Tucson AZ (USA) \\ email: kinman@noao.edu
}

\begin{abstract}
We have observed with UVES@VLT 15 field Blue Straggler Stars for which kinematical attribution to the Halo or Thick Disk is available. We present here the first results of our detailed chemical analysis.
\end{abstract}

Keywords. Stars: abundances, stars: blue stragglers, stars: fundamental parameters

\section{Our program: first results}

The sample of blue metal poor (BMP) nearby field stars collected by Preston et al. (1994) contains a large fraction of single-spectrum binaries (Preston \& Sneden (2000), PS00). At least one half of them are Blue Straggler Stars (BSS) formed by the McCrea mass transfer process, that mostly works in low density environments. While PS00, working on low $\mathrm{S} / \mathrm{N}$ spectra mainly aimed at deriving radial velocities, did not find any significant chemical peculiarity, subsequent dedicated studies revealed e.g., a large overabundance of Carbon and s-process elements in a few stars [for instance in CS294970030, see Sivarani et al. (2004) and Sneden et al. (2003)], suggesting that these stars have accreted processed material from their companions during the AGB phase.

The recent USNO-B catalog by Monet et al. (2003) gives proper motions for 49 stars of the PS00 sample, so we have been able to calculate the heliocentric space motions UVW, and separate Thick Disk and Halo stars on a kinematical basis (figure 1). We decided to study a fair fraction of them and derive detailed spectroscopic signatures in order i) to compare original Main Sequence and final abundances, to get information on the accreted material and the evolutionary phase(s) involved (RGB/AGB ?); ii) to find correlations with binary properties, and shed light on the formation mechanism; iii) to combine accurate abundances and abundance ratios with the space velocities and derive a better knowledge on the environment where these BMPs formed.

We observed 15 BMPs with UVES@VLT (program 72.D-0739), using a dichroic and covering the 326-451 and 665-1060 $\mathrm{nm}$ range at $\mathrm{R}=40000$ and $\mathrm{S} / \mathrm{N}>100$. Derivation of rotational velocities is in progress; we have already obtained abundances for the 7 stars with $V \sin i$ less than about $20 \mathrm{~km} \mathrm{~s}^{-1}$. Atmospheric parameters were derived in a full spectroscopic way, using the usual minimization methods to obtain temperature, gravity and microturbulent velocity. These will have to be validated using photometric information (V and JHK); special care will be used with C-rich stars, since colors may be affected. Results of this first stage of the analysis, limited to the blue portion of our spectra, are shown in the Table. 

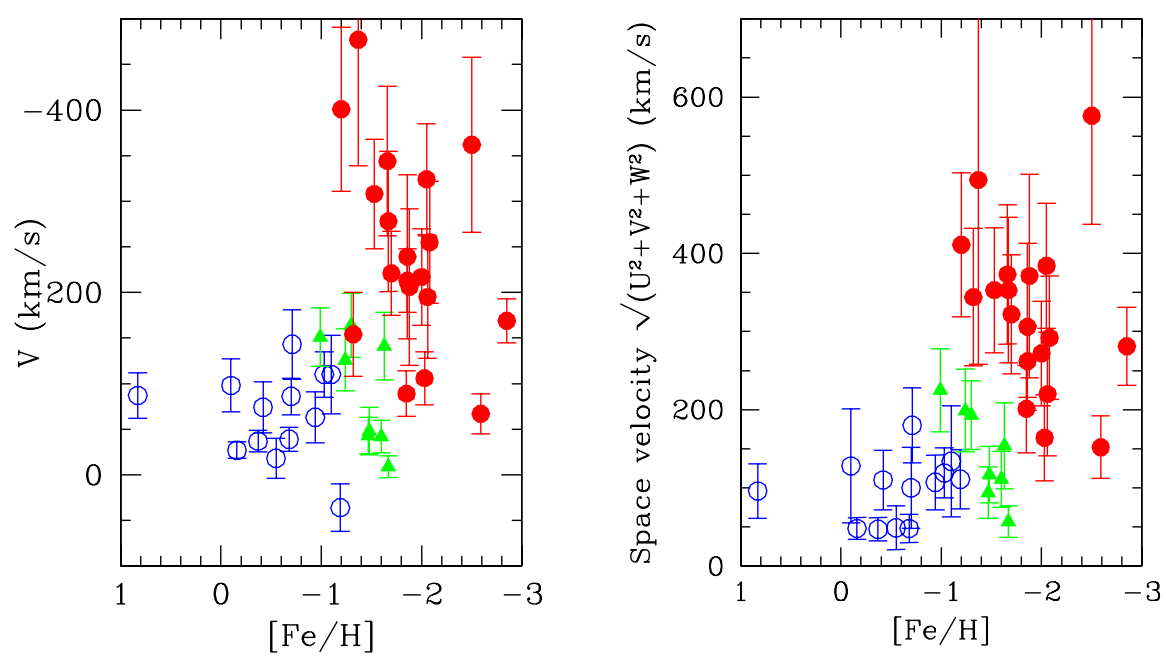

Figure 1. Rotation vector V(left) and space velocity (right) versus $[\mathrm{Fe} / \mathrm{H}]$ for the PS00 sample. Kinematically we can distinguish between Halo (filled dots) and Thick Disk (open dots) stars, with a few unclear cases (triangles).

Table 1. Atmospheric parameters (temperature, gravity, metallicity, microturbulent velocity), heliocentric radial velocities and iron abundances for the seven stars presently analysed

\begin{tabular}{lccccrccccl}
\hline Star name & $\mathrm{T}_{\text {eff }}$ & $\operatorname{logg}$ & {$[\mathrm{A} / \mathrm{H}]$} & $\mathrm{Vt}$ & $\mathrm{Vr}$ & {$[\mathrm{Fe} / \mathrm{H}] \mathrm{I}$} & $\mathrm{rms}$ & {$[\mathrm{Fe} / \mathrm{H}] \mathrm{II}$} & $\mathrm{rms}$ & Population \\
\hline CS22966-0054 & 7100 & 3.95 & -1.44 & 2.55 & -77.5 & -1.51 & 0.113 & -1.45 & 0.160 & Halo \\
CS22966-0043 & 7300 & 3.70 & -2.00 & 3.00 & -268.7 & -2.04 & 0.180 & -1.92 & & Halo \\
CS29497-0030 & 7150 & 4.28 & -2.29 & 1.85 & 44.2 & -2.40 & 0.150 & -2.43 & 0.159 & Halo \\
CS22956-0028 & 6940 & 4.20 & -2.29 & 1.80 & 41.3 & -2.31 & 0.163 & -2.34 & 0.043 & Halo \\
CS30339-0013 & 6900 & 4.05 & -0.69 & 2.00 & -30.5 & -0.77 & 0.112 & -0.76 & 0.101 & Thick Disk \\
CS22894-0043 & 7050 & 4.13 & -0.55 & 1.77 & -33.5 & -0.79 & 0.133 & -0.78 & 0.163 & Thick Disk \\
CS22955-0110 & 6650 & 3.75 & -1.15 & 1.60 & 2.4 & -1.27 & 0.170 & -1.27 & 0.123 & Thick Disk \\
\hline
\end{tabular}

Next steps in the analysis require an accurate elimination of telluric features in order to fully exploit our large wavelength coverage: several atomic lines of $\mathrm{C}, \mathrm{N}, \mathrm{O}, \mathrm{Na}$ are present in the red, contaminated part of the spectra. $\mathrm{N}$ and $\mathrm{C}$ abundances will also be obtained from spectrum synthesis of the $\mathrm{UV} \mathrm{NH}$ and $\mathrm{CN}$ bands and of $\mathrm{CH}$ band. $\mathrm{Li}$ appears (almost) absent from our spectra, but the visual impression has to be further checked.

Finally, we will compare our findings with existing literature, as for CS29497-0030, studied by Sivarani et al. (2004), Sneden et al. (2003) and Ivans et al. (this Symposium), or CS22956-0028 analysed by Lucatello (2004).

\section{References}

Lucatello, S. 2004, PhD Thesis, Padova University

Monet, D.G., et al. 2003, AJ 125, 984

Preston, G.W., Beers, T.C., \& Schectman, S.A. 1994, AJ 108, 583

Preston, G.W. \& Sneden, C. 2000, AJ 120, 1014 (PS00)

Sivarani, T., et al. 2004, A\&\&A 413, 1073

Sneden, C., Preston, G.W., \& Cowan J.J. 2003, ApJ 592, 504 